\title{
Resultados de un estudio de Contactos de pacientes con Tuberculosis en la Provincia de Toledo
}

\author{
Pilar Toledano Sierraa, Enriqueta Muñoz Platón ${ }^{b}$, Manuel Velasco Rodríguez ${ }^{\mathrm{b}}$, \\ Raúl Perea Rafael ${ }^{b}$, Ramón Orueta Sánchez ${ }^{\mathrm{c}}$
}

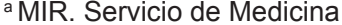
Interna, Hospital "Virgen de la Salud" de Toledo (España).

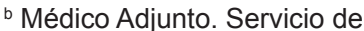
Medicina Preventiva, Hospital "Virgen de la Salud" de Toledo (España)

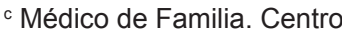
de Salud "Sillería" de Toledo (España).

Correspondencia: Ramón Orueta Sánchez, Centro de Salud "Sillería", C/ Silleria s/n, 45001-Toledo (España).

Correo electrónico:

roruetas@gmail.com

Recibido el 16 de noviembre de 2010.

Aceptado para su publicación el 22 de diciembre de 2010 .

\section{RESUMEN}

Objetivos. Conocer las características epidemiológicas de los contactos de personas con tuberculosis, la indicación de quimioprofilaxis primaria y/o secundaria en los mismos y el grado de seguimiento/perdidas del protocolo de estudio de los mismos.

Diseño. Descriptivo, transversal.

Emplazamiento. Área de Salud.

Participantes. Universo de contactos de pacientes con tuberculosis estudiados en un servicio de Medicina Preventiva de un Área de Salud durante los años 2007 y 2008.

Resultados. Fueron estudiados 419 contactos, los cuales tenían una edad media de 33,6 años; el $51,1 \%$ eran varones; el $17,4 \%$ eran inmigrantes con una estancia media en España de 5,3 años. Los factores de riesgo presentes en los mismos con mayor frecuencia fueron; tabaquismo (27\%), consumo de alcohol $(11,2 \%)$ y diabetes mellitus $(1,2 \%)$. El $8,8 \%$ refería contacto previo con enfermos con tuberculosis, el $11 \%$ haberse realizado prueba PPD con anterioridad, el $6,7 \%$ haber recibido la vacunación BCG y el 3,3\% haber realizado quimioprofilaxis con anterioridad. Únicamente el $1,7 \%$ de los contactos refería sintomatología compatible con tuberculosis.

Respecto al tipo de contacto, el $6,4 \%$ era laboral $(93,6 \%$ no laboral) y su intensidad diversa (intimo 10,9\%; más de $6 \mathrm{~h} / \mathrm{d} 32,9 \%$, diario pero menos de $6 \mathrm{~h} / \mathrm{d} 22,4 \%$ y más esporádico $33,7 \%$ ). Las localizaciones más frecuentes de la enfermedad en los casos fueron pulmonar $(78,4 \%)$, pleural (15\%) y linfática $(3,6 \%)$.

Tras la realización del protocolo de estudio de contactos, el $42,5 \%$ precisó quimioprofilaxis primaria, el $11 \%$ quimioprofilaxis secundaria y el $1 \%$ pasó al estudio de enfermedad tuberculosa. El total de pacientes que no completaron el protocolo fue del $1,7 \%$.

Conclusiones. El estudio pone en evidencia la necesidad del estudio de contactos de pacientes con tuberculosis, ya que un alto porcentaje de los mismos precisan quimioprofilaxis. El porcentaje de pacientes que no completó el protocolo fue inferior al recogido en otras publicaciones, lo que probablemente determinó el escaso porcentaje de contactos que pasó a enfermedad.

Palabras clave: Tuberculosis, Estudio Contactos, Medicina Preventiva.

\section{ABSTRACT}

Results of a Tuberculosis contacts study in the province of Toledo.

Objective. The objective of this study is to determine the epidemiological characteristics of contacts of persons diagnosed with tuberculosis, the indication for primary or/and secondary prophylaxis and the degree of compliance to the study protocol.

Design. Descriptive, Cross-sectional study.

Participants. People who had a tuberculosis contact and screened in a Preventive Medicine Department of the Toledo Health Area during the years 2007 and 2008.

Results. We recruited 419 contacts, with a mean age of 33.6 years of whom $51.1 \%$ were male and $17.4 \%$ were immigrants with an average stay of 5.3 years in Spain. The most frequent risk factors in our sample were: smoking $(27 \%)$, alcohol $(11.2 \%)$ and diabetes mellitus $(1.2 \%) ; 8.8 \%$ reported previous contact with TB patients, $11 \%$ having had prior PPD, $6.7 \%$ had received BCG vaccination and $3.3 \%$ reported having previously received chemoprophylaxis. Only $1.7 \%$ of the contacts reported symptoms compatible with tuberculosis.

Regarding the type of contact, $6.4 \%$ of them were contacts in the work place (or working contacts) $(93.6 \%$ were not related with the work place). Intensity varied (intimate 10.9\%; over 6 hours daily $32.9 \%$; daily but less than 6 hours a day $22.4 \%$ and more sporadic $33.7 \%$ ).

The most frequent sites of disease in those diagnosed with active disease were pulmonary (78.4\%) (pleural $15 \%)$ and lymph nodes $(3.6 \%)$

At the end of the contact tracing using our protocol $42.5 \%$ of the contacts required primary chemoprophylaxis, $11 \%$ secondary prophylaxis and $1 \%$ underwent further studies for active disease; $1.7 \%$.of patients did not complete the protocol.

Conclusions. This study demonstrates the need to screen tuberculosis contacts since a high percentage of them require chemoprophylaxis. The percentage of patients who did not complete the protocol in our study was inferior to that found in other publications, which probably determined the low percentage of contacts who were found to have active disease.

Keywords: Tuberculosis, Study contacts, Preventive Medicine. 


\section{INTRODUCCIÓN}

La tuberculosis, enfermedad infecciosa ocasionada principalmente por el mycobacterium tuberculosis, supone desde hace siglos un importante problema de salud a nivel mundial motivado tanto por su elevada incidencia y prevalencia como por su importante morbi-mortalidad ${ }^{1}$. Nuestro país no es ajeno a este problema y, aunque presenta tasas bajas de incidencia en relación a las cifras encontradas a nivel mundial y en los últimos años se observa un estancamiento de dichas tasas de incidencia con una discreta reducción de la tasa de tuberculosis pulmonar y un ligero aumento de su aparición en otras localizaciones, éstas continúan siendo superiores a las encontradas en otros países de nuestro entorno europeo ${ }^{2-3}$.

Como cualquier otra enfermedad de carácter transmisible, su paso a personas sanas se encuentra influido por distintos factores relacionados con el propio paciente enfermo, con factores relacionados con las personas sanas que conviven o se relacionan con dichos pacientes y con factores relacionados con el entorno en el que acontece dicho contacto, siendo la vía aérea la principal, aunque no única, vía de transmisión ${ }^{4-6}$.

Las estrategias fundamentales diseñadas para el control de la tuberculosis incluyen de forma prioritaria, lo cual es totalmente procedente, el diagnóstico precoz de los casos y la rápida instauración de un tratamiento adecuado en los mismos, pero ocupando también un lugar prioritario en dicha estrategia el estudio sistemático de los contactos de dichos pacientes y la instauración de tratamiento preventivo en aquellos contactos que lo requieran con el objetivo intermedio de cortar la cadena epidemiológica de transmisión y con el objetivo final de erradicar la enfermedad ${ }^{1,7,8}$. La necesidad y utilidad del estudio de los contactos viene determinada tanto por el diagnóstico precoz y tratamiento de los casos de enfermedad tuberculosa secundarios detectados a través dicha intervención como por el tratamiento preventivo en personas con infección tuberculosa latente (quimioprofilaxis secundaria) y en personas sanas (sin infección) con factores de riesgo (quimioprofilaxis primaria) $)^{7,9}$, existiendo estudios que han demostrado que las estrategias de estudio de contactos de pacientes con tuberculosis son intervenciones rentables en términos de coste-efectivi$\operatorname{dad}^{10-11}$.

En este contexto se presenta este trabajo que tiene como objetivos principales conocer las características epidemiológicas de los contactos de personas con tuberculosis, las cifras de indicación de quimioprofilaxis primaria y/o secundaria en los mismos y el grado de seguimiento/perdidas del protocolo de estudio de los mismos.

\section{MATERIAL Y MÉTODO}

Para obtener los datos necesarios para llegar a dar respuesta a los objetivos comentados en la introducción se diseñó un estudio de tipo descriptivo de corte transversal.

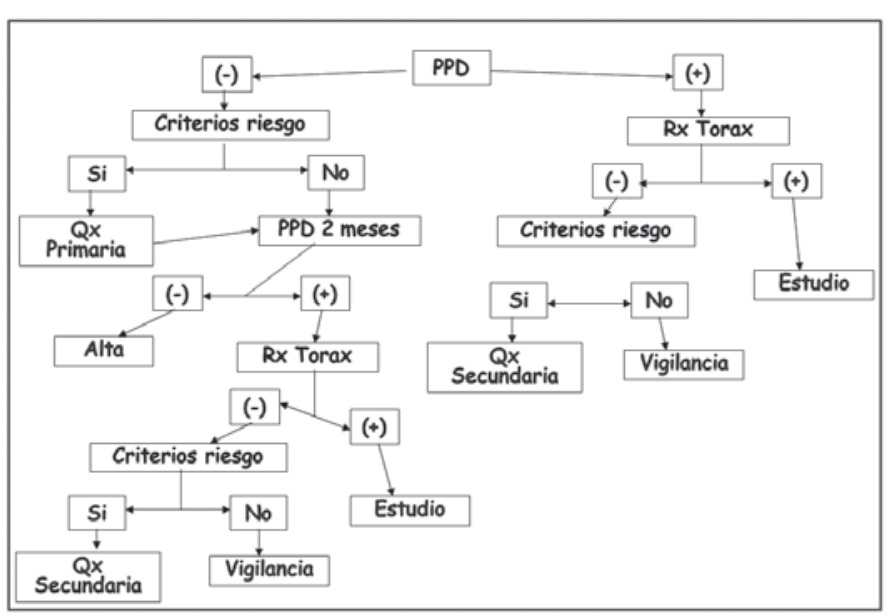

Figura 1. Agoritmo de actuación.

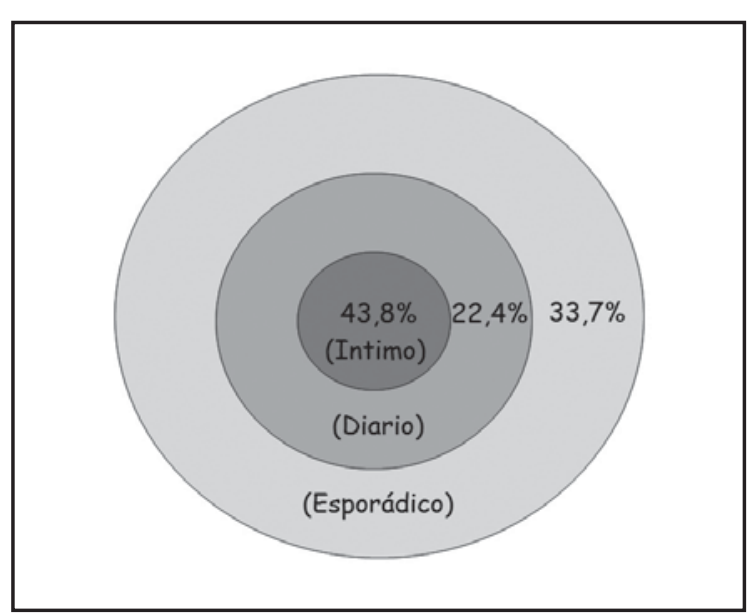

Figura 2. Intensidad del contacto. 


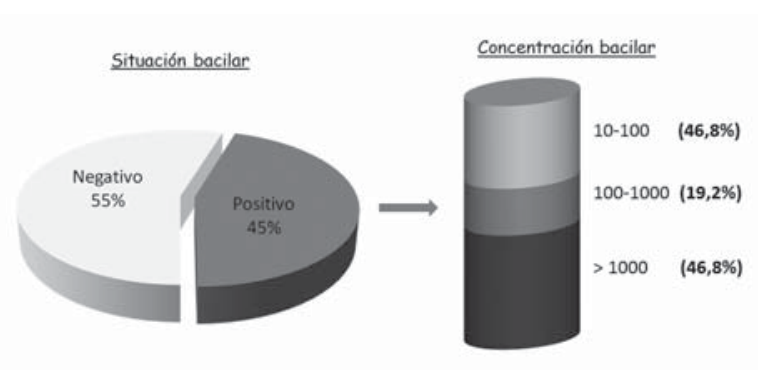

Figura 3. Situación bacilar caso.

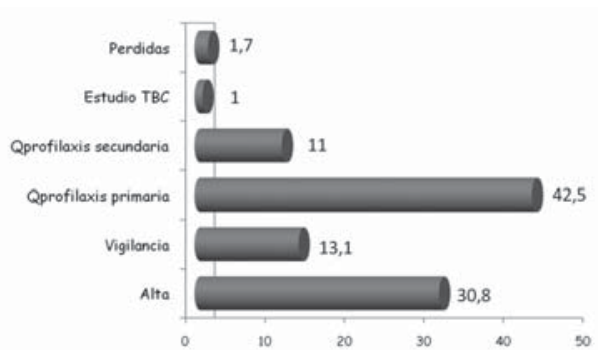

Figura 4. Actitud seguida.

\begin{tabular}{|c|c|}
\hline Situación & $\%$ \\
\hline $\begin{array}{l}\text { PPD inicial (-) con factores de riesgo } \\
\text { (Quimioprofilaxis primaria) }\end{array}$ & 42,5 \\
\hline $\begin{array}{l}\text { PPD inicial (-) sin factores de riesgo y PPD posterior (-) } \\
\text { (Alta) }\end{array}$ & 30,8 \\
\hline $\begin{array}{l}\text { PPD inicial (-) sin factores de riesgo y PPD posterior (-) con Rx }(-) \text { y con criterios de riesgo } \\
\text { (Quimioprofilaxis secundaria) }\end{array}$ & 1,9 \\
\hline $\begin{array}{l}\text { PPD inicial (-) sin factores de riesgo y PPD posterior }(+) \text { con } \operatorname{Rx}(-) \text { y sin criterios de riesgo } \\
\text { (Vigilancia) }\end{array}$ & 1,2 \\
\hline $\begin{array}{l}\text { PPD inicial (-) sin factores de riesgo y PPD posterior }(+) \text { con } \mathrm{Rx}(+) \\
\text { (Estudio de enfermedad) }\end{array}$ & - \\
\hline $\begin{array}{l}\text { PPD inicial (+) con } \mathrm{Rx}(-) \text { y con criterios de riesgo } \\
\text { (Quimioprofilaxis secundaria) }\end{array}$ & 9,1 \\
\hline $\begin{array}{l}\text { PPD inicial }(+) \text { con } \mathrm{Rx}(-) \text { y sin criterios de riesgo } \\
\text { (Vigilancia) }\end{array}$ & 11,9 \\
\hline $\begin{array}{l}\text { PPD inicial }(+) \text { con } \mathrm{Rx}(+) \\
\text { (Estudio de enfermedad) }\end{array}$ & 1 \\
\hline Pérdidas & 1,7 \\
\hline
\end{tabular}

Tabla 1. Actitud seguida.

La población diana del estudio fueron todos los contactos de pacientes tuberculosos atendidos durante los años 2007 y 2008 en el Servicio de Medicina Preventiva del Hospital "Virgen de la Salud" de Toledo; a dicho servicio fueron remitidos los contactos de todos los pacientes tuberculosos atendidos en los distintos servicios del mencionado hospital.

El estudio de los contactos se inició siguiendo el esquema recomendado de círculos concéntricos ${ }^{7}$. En una primera visita se les realizó una entrevista clínica donde se recogieron datos relativos a la persona atendida como contacto (sociodemográficos, factores de riesgo, sintomatología posiblemente relacionada con la enfermedad y antecedentes de enfermedad tuberculosa, vacunación BCG y/o prueba de tuberculina), datos relativos al paciente tuberculoso con el que convivían o mantenían el contacto (datos generales, localización, carácter bacilífero, etc.) y datos relativos al tipo de contacto mantenido con el paciente (relación con el paciente, intensidad del contacto, etc.). Además, a todos los pacientes se les practicó prueba de tuberculina (PPD), considerándose positiva una induración igual o superior a $5 \mathrm{~mm}$, con las excepciones a este respecto que 
marca la literatura ${ }^{12}$. Con los datos disponibles a través de la entrevista clínica y de los resultados de la prueba PPD, se actuó de forma individualizada, siguiendo los recomendaciones vigentes ${ }^{12-15}$ que aparecen recogidas en la figura 1 , valorándose la realización de radiología de tórax, indicación de quimioprofilaxis (primaria o secundaria) y/o estudio de posible enfermedad.

A continuación, se establecieron visitas sucesivas de seguimiento y control, en función del algoritmo de actuación anteriormente comentado, con el objetivo de completar el estudio, seguir la evolución y confirmar el cumplimiento terapéutico en caso de haberse pautado la realización de algún tipo de quimioprofilaxis.

Todos los datos obtenidos fueron introducidos en una base de datos creada para tal fin y analizados a través de herramientas de estadística descriptiva del paquete estadístico SPSS en su versión 15.0 para Windows.

\section{RESULTADOS}

Siendo 131 el número de pacientes diagnosticados de tuberculosis, fueron remitidos al servicio y estudiados un total de 419 contactos, lo que ofrece una media de 3,2 contactos por cada caso. Estos contactos tenían una edad media de 33,6 años (DE: 19,8 años) y el $51,1 \%$ eran varones. El $17,4 \%$ de los mismos eran inmigrantes, con una estancia media en España de 5,3 años (DE: 5,3 años), destacando como lugar de procedencia Marruecos (54,8\%), Rumania (35,6\%) y Ecuador (6,8\%).

Respecto a la presencia de factores de riesgo, destacaban, por su frecuencia, tabaco $(27 \%)$, alcohol $(11,2 \%)$ y diabetes mellitus $(1,2 \%)$, siendo el resto prácticamente testimoniales (inmunodepresión, desnutrición y silicosis con un $0,2 \%$ cada uno de los mismos).

Únicamente 7 de los contactos $(1,7 \%)$ presentaba algún síntoma compatible con padecer enfermedad tuberculosa. El 8,8\% refería algún contacto previo con enfermos tuberculosos, el $11 \%$ haberse realizado la prueba de tuberculina anteriormente, el 6,7\% estar vacunados con vacuna BCG y el $3,3 \%$ haber recibido quimioprofilaxis con anterioridad.

Los datos relativos a la intensidad del contacto aparecen recogidos en la figura 2 , siendo el $6,4 \%$ de carácter laboral y el $93,6 \%$ de carácter familiar y/o institucional (colegio, residencia, etc.).

Respecto a los datos de interés relativos a los ca- sos, destacar que las localizaciones más frecuentemente encontradas de la enfermedad en los mismos fue la localización pulmonar $(78,4 \%)$, seguida de la pleural (15\%) y de la linfática $(3,6 \%)$. Respecto a la situación bacilífera de los casos, los datos aparecen recogidos en la figura 3 .

La actitud seguida en los distintos contactos, de acuerdo con el algoritmo de estudio y tratamiento comentado en el apartado de material y métodos, aparece recogida en la tabla 1 (La figura 4 recoge estos datos de forma agrupada). Durante el periodo de seguimiento, únicamente 7 personas $(1,7 \%)$ no completaron el protocolo de estudio y/o tratamiento propuesto, siendo considerados como pérdidas.

\section{DISCUSIÓN}

Distintas recomendaciones, tanto nacionales como internacionales, ponen de manifiesto de forma rotunda la utilidad y la necesidad del estudio de contactos de pacientes tuberculosos $7,9,15-18$, existiendo así mismo protocolos de actuación que detallan las acciones y/o tratamiento a realizar en dichas personas en función de su situación ${ }^{12-18}$. Pese a ello, distintos trabajos publicados en nuestro entorno ponen en evidencia que con frecuencia no se obtienen las coberturas deseadas ${ }^{19-23}$, lo que puede condicionar sus resultados en términos de efectividad para erradicar el proceso y de eficiencia de la intervención $n^{8,11}$.

El número de contactos estudiados por cada caso de tuberculosis es ligeramente inferior al encontrado en otras publicaciones realizadas en contactos de nuestro entorno, si bien las características sociodemográficas generales encontradas son similares a las de los mismos ${ }^{19-21,24-29}$, lo que nos hace pensar en la existencia de una posible ligera hipocaptación de los contactos (no es posible conocer el número de contactos remitidos al servicio que no acudieron al mismo), pero sin sesgos en la misma, por lo que, en caso de existir dicha hipocaptación, ésta no haría perder validez a los resultados de la investigación.

Diferentes investigaciones han demostrado que la presencia de tuberculosis de localización pulmonar en los pacientes y su condición de bacilíferos influyen de forma directa en la transmisión del proceso a sus contactos ${ }^{21,25,26,30-32}$; en este sentido, nuestro porcentaje de contactos que procedían de enfermos con tuberculosis pulmonar $(78,4 \%)$ es similar al de otras publicaciones, si bien el porcentaje de contactos procedentes de pacientes bacilíferos $(45,2 \%)$ es algo inferior al encontrado en dichos estudios $21,25,28$. 
En la misma línea, pero en sentido contrario, el porcentaje de contactos íntimos/intensos encontrados en nuestro estudio $(43,8 \%)$ es superior al encontrado en los mencionados trabajos ${ }^{21,25,28}$, existiendo estudios que ponen en evidencia la relación directa entre la intensidad del contacto y la posibilidad de transmisión de la infección tuberculosa ${ }^{30-34}$.

El objetivo de la quimioprofilaxis primaria es evitar la infección tuberculosa en personas sanas expuestas a un foco contagioso, existiendo criterios claros de cuándo tratar y cuándo no tratar a dichas personas en función de la presencia o no de factores de riesgo $0^{7,15,35,36}$. Cabe destacar que un alto porcentaje de los contactos estudiados $(42,5 \%)$ precisó de quimioprofilaxis primaria al no tener evidencias de infección/enfermedad pero presentar algún factor de riesgo incluido en las recomendaciones, destacando la edad como principal factor presente.

Las personas con infección tuberculosa latente tienen una probabilidad de desarrollar la enfermedad del $5-10 \%$, con predominio en personas con edades extremas, con deterioro inmunológico y/o inmigrantes procedentes de países con alta endemia tuberculosa, y ello determina la importancia de su abordaje terapéutico (quimioprofilaxis secundaria $)^{37-38}$. El porcentaje de contactos encontrados en nuestro estudio con infección tuberculosa latente $(24,1 \%)$, y dentro de los mismos los que presentan factores de riesgo que indican la necesidad de quimioprofilaxis secundaria (11\%), es inferior a la encontrada en otros estudios realizados en nuestro país $^{19,21,22,24-28,30,32}$. En la misma línea, el porcentaje de contactos con posible enfermedad tuberculosa en el momento del estudio (1\%) es inferior al encontrado en los mismos estudios.

Por último, el número de pérdidas a lo largo del estudio es muy bajo $(1,7 \%)$ en comparación con otros estudios que aportan este dato $21,22,24,32,39$, dato que como ya se ha comentado es vital para mantener la eficacia y eficiencia de la intervención y probablemente sea, al menos en parte, responsable del bajo porcentaje de casos de enfermedad encontrados en los mismos.

Por todo ello, concluimos que los resultados ponen en evidencia la necesidad del estudio de contactos de pacientes con tuberculosis, ya que un alto porcentaje de los mismos precisa quimioprofilaxis, y la importancia de obtener cifras elevadas de seguimiento del programa, ya que esto puede limitar el paso de dichas personas a enfermedad tuberculosa.

\section{BIBLIOGRAFÍA}

1. WHO. Global tuberculosis control: epidemiology, strategy, financing. WHO Report 2009. Geneva: World Health Organization; 2009 (WHO/HTM/TB/2009.411).

2. Rodríguez E, Hernández G, Díaz O, Tello O. Casos de tuberculosis declarados a la Red Nacional de Vigilancia Epidemiológica. España, 2007. Boletín Epidemiológico Semanal. 2008; 16(20):229-32.

3. Ríos M, Monleón-Getino T. A graphical study of tuberculosis incidente and trenes in the WHO's European region (20002006) Eur J Epidemiol. 2009; 24:381-7.

4. Riley RL. Disease transmission and contagion control. Am Rev Resp Dis. 1982; 125:16-9.

5. Rodrigo T, Cayla JA, Garcia P, Galdós H, Jansa JM, Miranda P. Charasteristics of tuberculosis patients who generate secondary cases. Int J Tuber Lung Dis. 1997; 1(4):352-7.

6. Lienhardt C. From exposure to disease: the role of environmental factors in susceptibility to and development of tuberculosis. Epidemiol Rev. 2001; 23(2):288-301.

7. Grupo de expertos en tuberculosis y grupo de trabajo de las Comunidades Autónomas. Plan para la prevención y control de la tuberculosis en España. Madrid: Ministerio de Sanidad y Consumo; 2008. Disponible en: http://www.msc.es/profesionales/saludPublica/prevPromocion/docs/planTuberculosis.pdf.

8. Centers for Disease Control and Prevention (CDC). Advisory Council for the Elimination of Tuberculosis. Tuberculosis elimination revisited: obstacles, opportunities, and a reviewed commitment. MMWR. 1999; 48(RR-9):1-13.

9. Pitman R, Jarman B, Coker R. Tuberculosis transmission and the impact of intervention on the incidence of infection. Int J Tuberc Lung Dis. 2002; 6:485-91.

10. Macintyre CR, Plant AJ, Hendrie D. The cost-effectiveness of evidence-based guidelines and practice for screening and prevention of tuberculosis. Health Econ. 2000; 9:411-21.

11. Dasgupta K, Schwartzman K, Marchand R, Tennenbaum TM, Brassard P, Menzies D. Comparasion of cost-effectiveness of tuberculosis screnning of close contacts and foreignborn populations. Am J Crit Med Care. 2000; 162:2079-86.

12. García MJ, Rigueiro MT, Casariego E, Corredoira JC, Varela J, García JF. Tuberculosis. Guias Fisterra. 2003; 3(25). Disponible en: http.//wwww.fisterra.com.

13. Centers for Disease Control and Prevention (CDC). Guidelines for the investigation of contact of persons with infectious tuberculosis. MMWR. 2005; 54(RR15):1-37.

14. Gomez Garcia M, Rodriguez Barrientos R. Estudio de contactos de enfermos de tuberculosis. AMF. 2006; 2(8):464-9.

15. Teruel F, Castilla J, Hueto J. Abordaje de la tuberculosis en Atención Primaria. Estudio de contactos. An Sist Sanit Navar. 2007; 30 (supl2):S87-S98.

16. Rieder HL. Interventions for TB control and elimination. Paris: International Union Against Tuberculosis and Lung Disease; 2002.

17. Enarson D, Arnadottir T, Trebucq A. Management of tuberculosis: a guide for low income countries, 5th ed. Paris: International Union Against Tuberculosis and Lung Disease; 2000.

18. Stop TB Partnership, Child TB Subgroup, WHO. Guidance for national tuberculosis programmes on the management of tuberculosis in children. WHO/HTM/TB/2006.371.

19. Solsona-Peiró J. Estudio convencional de contactos versus epidemiología molecular en una zona de alta prevalencia de tuberculosis. Resultados preliminares. Rev Esp Sanid Penit. 1999; 1:149-51.

20. Alsedá M, Godoy P. Factores del enfermo tuberculoso asociados al estudio de contactos. Rev Clin Esp. 2004; 204(1):3-8. 
21. Salinas C, Capelastegui A, Altube L, España PP, Díez R, Oribe $M$, et al. Incidencia longitudinal de la tuberculosis en una cohorte de contactos: factores asociados a la enfermedad. Arch Bronconeumol. 2007; 43(6):317-23.

22. Palomo M, Rodríguez C, Eyerbe T. Un estudio de contactos de tuberculosis en inmigrantes marroquíes. Rev Esp Salud Pública. 1996; 70:345-9.

23. Rodrigo T, Caylá JA. Efectividad de los programas de control de la tuberculosis en España. Med Clin (Barc). 2003; 121(10):375-7.

24. Del Castillo D, Peñafiel M, Alvarez F, Soto JG, Sánchez J, Calderón E, et al. Estudio de 2.207 contactos de enfermos con tuberculosis. Neomosur. 1999; 11(3):27-34.

25. Vidal R, Miravitlles M, Caylá JA, Torella M, Martín M, De Gracia J. Estudio del contagio de 3071 contactos familiares de enfermos con tuberculosis. Med Clin (Barc) 1997; 108:361-5

26. Alsedá M, Godoy P. Factores asociados a la enfermedad tuberculosa latente en los contactos de pacientes afectados. Gac Sanit. 2004; 18(2):101-7.

27. Alsedá M, Godoy P. El tamaño de la reacción tuberculínica en contactos de pacientes tuberculosos. Arch Bronconeumol. 2007; 43(3):161-4.

28. Alsedá M, Godoy P. Estudio de contactos de enfermos tuberculosos en un área semiurbana. Enferm Infecc Microbiol Clin. 2003; 21(6):281-6.

29. Exojo F, Ruiz MJ, Garcia C, García J. Estudio de contactos de tuberculosis. Área sanitaria de Toledo y Mancha-Centro. Libro de ponencias. XI Congreso de Atención Primaria de Castilla-La Mancha, Toledo, 2010.

30. Reichler MR, Reves R, Bur S, Thompson V, Mangura BT, Ford $\mathrm{J}$ et al. Evaluation of investigations conducted to detect and prevent transmission of tuberculosis. JAMA. 2002; 287(8):991-5.
31. Rathi SK, Akhtar S, Rahbar MH, Azam SI. Prevalence and risk factors associated with tuberculin skin test positivity among household contacts of smear-positive pulmonary tuberculosis cases in Umerkot, Pakistan. Int $\mathrm{J}$ Tuberc Lung Dis. 2002; 6(10):851-7.

32. Del Castillo D, Peñafiel M, Álvarez F, Soto JG, Calderón $\mathrm{E}$, Toral J, et al. Investigation of Tuberculosis Contacts in a Nonhospital Pneumology Practice. Eur J Clin Microbiol Infect Dis. 1999; 18:790-5.

33. Carvalho A, DeRiemer K, Nunes Z, Martins M, Comelli M, Marinoni A et al. Transmission of Mycobacterium tuberculosis to Contacts of HIV-infected Tuberculosis Patients. Am J Respir Crit Care Med. 2001; 164:2166-71.

34. Lienhardt C, Fielding K, Sillah J, Tunkara A, Donkor S, Manneh $\mathrm{K}$ et al. Risk Factors for Tuberculosis Infection in Sub-Saharan Africa. A Contact Study in The Gambia. Am J Respir Crit Care Med. 2003; 168:448-55.

35. Tortola D, Monedero MJ, Garcés FJ. Tuberculosis pulmonary en inmunocompetentes y en VIH positivos. Informe Técnico de la SVMFIC. Disponible en: htpp://www.svmfyc. rg/Revista/05/tuberculosis.asp.

36. Grupo de trabajo del área TIR de SEPAR. Recomendaciones SEPAR. Normativa sobre la prevención de la tuberculosis. Arch Bronconeumol. 2002; 38(9):441-51.

37. Moreno S, Cobo J. Las múltiples caras del control de la tuberculosis. Enferm Infecc Microbiol Clin. 2003; 21:279-80.

38. KHan K, Muennig P, Behta M, Graff J. Global drug-resistance patterns and the management of latent tuberculosis infection in immigrants to the United States. N Engl J Med. 2002; 347:1850-9.

39. Alvarez-Castillo MC, Cano Escudero S, Taveira Jiménez JA. Microepidemias de tuberculosis en centros escolares. ¿Cómo seleccionar los contactos? Gac Sanit. 2007; 21(6):465-70. 